\title{
Effect of Age and Season on Enteritis and Antibiotic Sensitivity Test of $E$. coli Isolated from Infected Chickens in Odisha, India
}

\author{
Mayadevi Majhi ${ }^{1}$, Jasmine Pamia ${ }^{1 *}$, Susen Kumar Panda ${ }^{1}$, \\ Lipismita Samal ${ }^{2}$ and Rajashree Mishra ${ }^{3}$
}

\author{
${ }^{1}$ Divsion of Veterinary Pathology, C.V.Sc. and A.H., OUAT, Bhubaneswar \\ ${ }^{2}$ Divsion of Poultry Science, C.V.Sc. and A.H., OUAT, Bhubaneswar \\ ${ }^{3}$ Divsion of Veterinary Microbiology, C.V.Sc. and A.H., OUAT, Bhubaneswar \\ College of Veterinary Science and Animal Husbandry \\ Orissa University of Agriculture and Technology \\ Bhubaneswar, India \\ *Corresponding author
}

\section{A B S T R A C T}

\section{Keywords}

Season, Age,

Enteritis,

Colibacillosis,

Fowl hematology

Article Info

Accepted:

16 February 2018

Available Online:

10 March 2018
The incidence of enteritis in chicken was assessed in two organized farms of Odisha over a period of one and half years from January 2016 to July 2017. Detailed information about the farms like location of the farms, genotype of birds, age of birds, mortality, and cause of death were collected and noted. Blood samples from 120 numbers of enteritis affected birds and 10 numbers of apparently healthy birds of the same flock were examined for hematological parameters. The incidence of enteritis was found to be $28 \%$ from the necropsy of birds from the two organized farms. It was concluded that prevalence of enteritis in different farms was more during rainy (57\%) than summer (39\%) and winter (19\%) seasons. The prevalence of enteritis in birds of $0-14$ week's age group (51\%) was more susceptible to the disease than older age groups (4\%). The average values of total leucocytes count (thousands/ cubic $\mathrm{mm}$ ) and heterophils (\%) were significantly higher in the affected birds. It was revealed by etiological diagnosis of enteritis that $E$. coli has the highest involvement $(80 \%)$ than nonspecific causes $(17 \%)$ and coccidiosis $(3 \%)$. The antibiotic sensitivity test of avian colibacillosis showed highest sensitivity for Gentamicin.

\section{Introduction}

The basic role of poultry production is turning feed stuffs into meat. Thus healthy enteric system of growing poultry is a vital component for the success of poultry production. Any disturbance of digestive system is mostly accompanied with high economic losses due to poor performance, increased mortality rates, decreased weight gain, increased feed conversion rates and increased medication costs. Several pathogens (bacteria, viruses and parasites) are implicated as possible cause of enteric disorders either alone or in synergy with other microorganisms, or with non-infectious causes such 
as feed and /or management related factors. In the environment of hatchery and chicken houses, the intestine of day-old chicks is immediately colonized by many species of Gram negative and positive bacteria, aerobes and anaerobes (Karpinska et al., 2001). The gastrointestinal (GI) tract has the most extensive exposed surface in the body and is constantly exposed to a wide variety of potentially harmful substances. The GI tract must selectively allow the nutrients to cross the intestinal wall into the body while preventing the deleterious components of the diet from crossing the intestinal barrier (Korver, 2006). Owing to extensive scientific and technical transformation in poultry production, birds are becoming susceptible to various digestive disordersamong which, enteritis is a major problem.Enteritis in chicken pose a threat to intestinal health and can contribute to poor feed efficiency, decreased weight gain with an overall increased mortality, and increased risk of contamination of poultry products for human consumption.Enteritis means inflammation of intestine. It causes increased motility of the gut and thus, decreased absorption with increased secretion. However diseases particularly enteritis acts as a major hindrance in the progress of poultry farming. Under field conditions, it is difficult to determine the true cause of enteritis. Diagnosis of enteritis can be challenging and involves a comprehensive analysis of flock history and condemnation records, clinical signs, gross and histopathology, culture and response to treatment and molecular techniques. So diagnosis of bacterial enteritis should be done at early stage in order to minimize the heavy loss to the farmers. As there is serious threat to gastrointestinal health of poultry by enteric infections, this study involves investigating colibacillosis giving emphasis to season and age of the birds so as to alleviate the risk of farmers in advance.

\section{Materials and Methods}

The present study involved two organized farms of Odisha for period of one and half years from January 2016 to July 20117. Government farms like Poultry Breeding Farm of AICRP on poultry improvement (College poultry farm) and IPDP, Laxmisagar, Bhubaneswar and others birds presented from private poultry farms to the department of Veterinary pathology, C.V.Sc and A.H were included in the study.

\section{Epidemiological study}

Retrospective data regarding the history, season, genotype of birds, total incidences, age of birds, occurrence of disease, age wise mortality rate etc. were retrieved from the farm records of Poultry Breeding Farm of AICRP on poultry improvement (College poultry farm) and IPDP, Laxmisagar, Bhubaneswar.

\section{Hematological study}

Blood samples from 120 numbers of enteritis affected birds and 10 numbers of apparently healthy birds were examined for hematological parameters like Hemoglobin, Packed Cell Volume (PCV), Total Erythrocyte Count (TEC), Total Leucocytes Count (TLC), and Differential Count (DC).EDTA vials were used for collection of blood for hematological purpose. Blood (2$3 \mathrm{ml}$ ) was drawn from wing vein of birds into sterile syringe and poured into the EDTA vial. Then hematological parameters were estimated. Hemoglobin concentration in the blood was determined by Sahli's acid haematin method by the help of Sahli's haemoglobinometer. TEC and TLC were counted by haemocytometer using Natt and Herrick's diluting fluid (Natt - Herrick, 1952 Technique). For differential count in chicken blood smear was prepared. Then staining was 
done with a special stain known as WrightGiemsa stain, a type of modified Giemsa stain.

\section{Microbial examination}

Impression smears from intestine and intestinal swabs were collected aseptically from fresh carcasses which died due to enteritis for Gram's staining and isolation of causative bacteria respectively.

\section{Antibiotic sensitivity test for avian $E$. coli}

Antibiotic sensitivity test for avian colibacillosis was carried out by Kirby-Bauer disc diffusion technique following CLSI (Clinical and Laboratory Standards Institute) guidelines (Wayne, 2011). The inoculum was prepared by suspending the isolate with normal saline equal to turbidity of 0.5 McFarland turbidity standards $\left(10^{6}\right.$ $\mathrm{CFU} / \mathrm{mL}$ ).And with the help of sterile cotton swab the culture was evenly applied on the surface of the Mueller Hinton Agar (MHA) plates.Antibiotic discs (HiMedia, Mumbai) were placed on the surface of the agar using sterilized forceps. The discs were gently pressed onto the surface of the agar using flame sterilized forceps. The inoculated plates were carefully inverted and incubated for 24 hours at $37^{\circ} \mathrm{C}$ and the result was interpreted as per CLSI standard.

\section{Statistical analysis}

The hematological profiles of both healthy and infected birds were subjected to t-test to know the significance level of different parameters and were declared significant at $\mathrm{P} \leq 0.05$.

\section{Results and Discussion}

The incidence of enteritis in chicken was assessed in two organized farms of Odisha over a period of one and half years from
January 2016 to July 2017. Government farms like poultry breeding farm of AICRP on poultry improvement (College poultry farm) and IPDP, Laxmisagar, Bhubaneswar and others birds presented from private poultry farms to the department of Veterinary pathology were included in the study.

\section{Incidence of enteritis}

The incidence of enteritis was found to be $28 \%$ from the necropsy of birds from the two organized farms and other private farms. A total of 1549 number of birds were presented for postmortem examination, out of which 448 numbers of birds exhibited lesions of enteritis. It was found that the birds presented from IPDP farms were more affected (68\%) than the birds received from Poultry Breeding Farm (22 \%) of AICRP on poultry improvement of the college campus.

\section{Season wise study}

It was concluded that prevalence of enteritis in different farms was more during rainy $(57 \%)$ than summer $(39 \%)$ and winter $(19 \%)$ seasons (Fig. 1). This might be due to the fact that microbial proliferation increases radically in damp and moist environment. It may be noted that the number of affected cases were more in winter (October to February) i.e., 796 and summer (March to June) i.e., 702 but mortality percentage of the birds due to enteritis was not as high as compared to rainy season (July to September) with presentation of only 51 birds but 29 of them were affected with enteritis. Rahman et al., (2004) recorded colibacillosis in all three seasons of the year with significantly high rate during summer season at BRAC Poultry Disease Diagnostic Centre, Gazipur, Bangladesh. Hermans and Morgan (2007) observed high disease prevalence during the months of October to February in United Kingdom. Balasubramaniam and Dorairajan (2009) analyzed the influence of season on the 
mortality pattern of poultry in Namakkal belt, Tamil Nadu and found high Incidence of chronic respiratory disease and colibacillosis with no influence of season on their occurrence. They also observed the high incidence of necrotic enteritis in winter $(2.53 \%)$ and rainy season $(1.84 \%)$ as compared to summer season. Datta et al., (2013) studied on the epidemiology of enteritis in broiler chickens in Haryana and reported highest prevalence of enteritis in rainy and winter seasons.

\section{Age wise variation}

The prevalence of enteritis in different farms revealed that birds of $0-14$ week's age group $(51 \%)$ were more susceptible to the disease than older age groups (Fig. 2). This may be due to resistance of adult birds and young birds naturally susceptible to infectious conditions. Out of 448 birds affected with enteritis, 228 numbers of birds of age group 0-14 weeks were found affected with enteritis followed by 131 birds of 15-28 weeks, and rest 83 birds were more than 29 weeks of age group. Rahman et al., (2004) found widely prevalence of avian colibacillosis in all age groups of chickens of Gazipur, Bangladesh with especially high prevalence rate in adult layers. Daryoush et al., (2011) evaluated the epidemiological prevalence of the various kinds of enteritis in association with age, strain and sex in Azerbaijan Province of Iran. One-sample chi-square test, revealed that the highest rate of occurrence $(78.57 \%)$, belonged to broilers $(\mathrm{p}<0.01)$. It was observed greatly in 4-6 weeks of age $(\mathrm{p}<0.01)$. Datta et al., (2013) recorded maximum cases of enteritisin 29-35 days of age with $30.35 \%$ prevalence from 481 samples of broiler birds at Haryana.

\section{Etiological study}

It was revealed by etiological diagnosis of enteritis that $E$. coli has the highest involvement $(80 \%)$ than nonspecific causes
(17\%) and coccidiosis (3\%) (Fig. 3). From 448 enteritis cases it was found that 359 cases were positive for colibacillosis. Nonspecific causes included primarily cases of anemia, weakness, debility along with enteritis. Islam et al., (2004) isolated E. coli from broiler birds affected with characteristic lesions of omphalitis, yolk sac infection, fibrino pericarditis, perihepatitis, hemorrhagic enteritis and accumulation of excess pericardial and peritoneal fluid. Rahman et al (2007) examined a total 8169 dead chickens (2960 chicks, 1083 grower and 4126 adults) from Gazipur, Bangladesh. They collected related samples like liver, spleen and intestine and then cultured on different bacteriological agar media and found salmonellosis in $53.90 \%$ of chicks (group 1) followed by omphalitis in $28.42 \%$, colibacillosis in $13.36 \%$, mycoplasmosis in $2.55 \%$, necrotic enteritis in $1.18 \%$ and infectious coryza in $0.59 \%$.

The bacterial diseases salmonellosis, colibacillosis, infectious coryza and necrotic enteritis were detected in 55.96, 11.93, 29.91 and $2.20 \%$ of group 2 (growers), respectively. Bonia et al., (2010) studied on the incidence and pathological conditions in chicken of Kalinga Brown breed in Guwahati, Assam. They screened total of 120 chicken of Kalinga Brown breed for the prevalence of various disease conditions and isolated E. coli in all the affected cases.

Bhalerao et al., (2011) studied the incidence and pathological lesions of $E$. coli infection in natural cases of poultry carcasses in Haryana. They isolated E. coli from blood and liver samples in $86.6 \%$ cases. Dutta et al., (2011) detected and characterized Shiga toxin producing E. coli (STEC) and enteropathogenic E. coli (EPEC) from rectal swabs, intestinal contents, heart blood and spleen of 19 poultry birds of Aizawl, Mizoram that died due to acute diarrhea. 


\section{Hematology}

The average values of TLC (thousands/ cubic $\mathrm{mm}$ ) and heterophils (\%) were significantly higher in the affected birds than apparently healthy birds (Table 1). There was no significant difference between the average hemoglobin value of the affected birds $(10.40$ $\mathrm{g} / \mathrm{dl})$ and apparently healthy birds (10.17 $\mathrm{g} / \mathrm{dl}$ ). The average TLC in the affected birds (46667 thousands/ cubic $\mathrm{mm}$ ) showed significant increase in comparison to apparently healthy birds (20000 thousands/ cubic $\mathrm{mm}$ ). The average values of PCV\% showed no significant difference between the affected birds (36) and apparently healthy birds (38). There was no significant difference between the average TEC value of the affected birds (3.40 million per cubic $\mathrm{mm}$ ) and apparently healthy birds (3.10 million per cubic $\mathrm{mm}$ ). Average heterophils $\%$ of the affected birds $(48.67 \%$ was found to be significantly higher than apparently healthy birds $(25.00 \%)$. The average value of the lymphocytes \% of affected birds (47.00) showed significantly lower value in comparison to the values (69.00) of apparently healthy birds. The average value of monocytes $\%$ of affected birds (2.0) showed significantly lower value in comparison to the values (8.33) of apparently healthy birds. Average value of the eosinophils \% (1.67) and basophils \% (1.33) showed a lower value than the corresponding values (2.00 and 1.00) of the apparently healthy birds. Arukha (2015) found that the average values of haemoglobin, TLC and DC were significantly higher in the affected birds than apparently healthy birds of the same flock. Average heterophils $\%$ of the affected birds $(67.65 \%)$ was found to be significantly higher than apparently healthy birds (35.57\%). Mishra (2014) revealed that the hemoglobin, TEC, PCV and heterophils percentage were increased due to heat stress in chicken.Guabiraba and Schouler (2015) observed that heterophils would arrive very quickly at the infected sites probably due to a robust production of CXC chemokines during bacterial infection.

Table.1 Comparative hematological profile of enteritis affected birds with healthy birds

\begin{tabular}{|l|c|c|c|c|}
\hline Attributes & $\begin{array}{c}\text { Enteritis } \\
\text { affected birds }\end{array}$ & $\begin{array}{c}\text { Healthy } \\
\text { birds }\end{array}$ & T value & P value \\
\hline Hb (g/dl) & $10.40 \pm 0.64$ & $10.17 \pm 0.73$ & 0.241 & 0.822 \\
\hline PCV (\%) & $36.33 \pm 0.88$ & $38.00 \pm 1.15$ & -1.147 & 0.315 \\
\hline TEC (millions/ cubic mm) & $3.40 \pm 0.12$ & $3.10 \pm 0.06$ & 2.324 & 0.081 \\
\hline TLC (thousands/ cubic mm) & $46667^{\mathrm{a}} \pm 2186$ & $20000^{\mathrm{b}} \pm 577$ & 11.795 & 0.000 \\
\hline Heterophils (\%) & $48.67^{\mathrm{a}} \pm 3.48$ & $25.00^{\mathrm{b}} \pm 1.73$ & 6.088 & 0.004 \\
\hline Lymphocytes\% & $47.00^{\mathrm{b}} \pm 2.65$ & $69.00^{\mathrm{a}} \pm 3.21$ & -5.284 & 0.006 \\
\hline Monocytes \% & $2.00^{\mathrm{b}} \pm 0.00$ & $8.33^{\mathrm{a}} \pm 2.03$ & -3.124 & 0.035 \\
\hline Eosinophil \% & $1.67 \pm 0.33$ & $2.00 \pm 0.58$ & -0.500 & 0.643 \\
\hline Basophil \% & $1.33 \pm 0.33$ & $1.00 \pm 0.29$ & 0.756 & 0.492 \\
\hline \multicolumn{2}{|c|}{${ }^{\mathrm{a}, \mathrm{b}}$ Means bearing different superscripts in a row differ significantly } & & \\
\hline
\end{tabular}


Table.2 Antibiotic sensitivity profile of chicken colibacillosis

\begin{tabular}{|l|c|c|c|}
\hline \multicolumn{1}{|c|}{ Antibiotic } & Total no. of samples & $\begin{array}{c}\text { No. of susceptible } \\
\text { samples }\end{array}$ & Sensitivity \% \\
\hline Gentamicin & 30 & 27 & 90 \\
\hline Amikacin & 30 & 24 & 80 \\
\hline Colistin & 30 & 21 & 70 \\
\hline Cefotaxime & 30 & 18 & 60 \\
\hline Enrofloxacin & 30 & 15 & 50 \\
\hline Ciprofloxacin & 30 & 12 & 40 \\
\hline Ampicillin & 30 & 6 & 20 \\
\hline Chloramphenicol & 30 & 6 & 20 \\
\hline Lincomycin & 30 & 3 & 10 \\
\hline
\end{tabular}

Fig.1 Season wise mortality

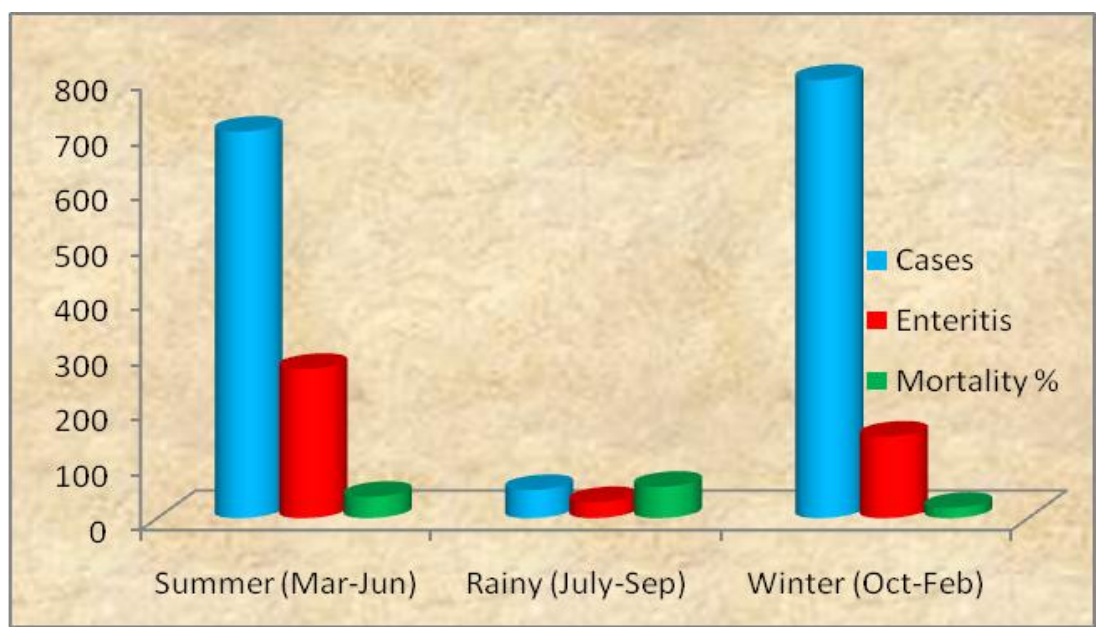

Fig.2 Age wise mortality

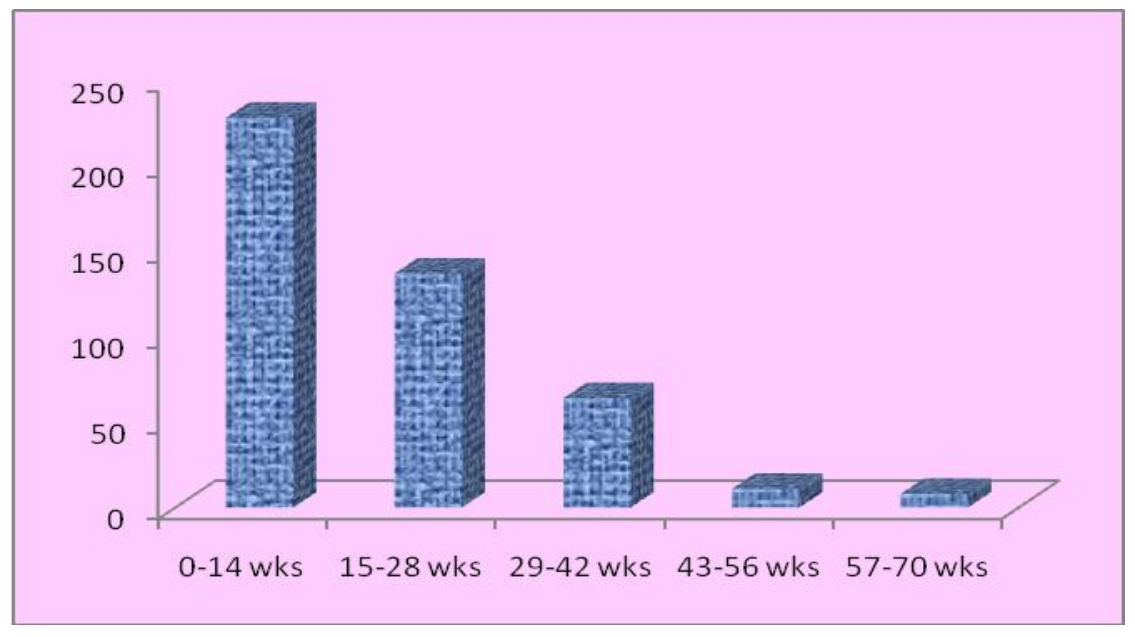


Fig.3 Etiological classification of enteritis

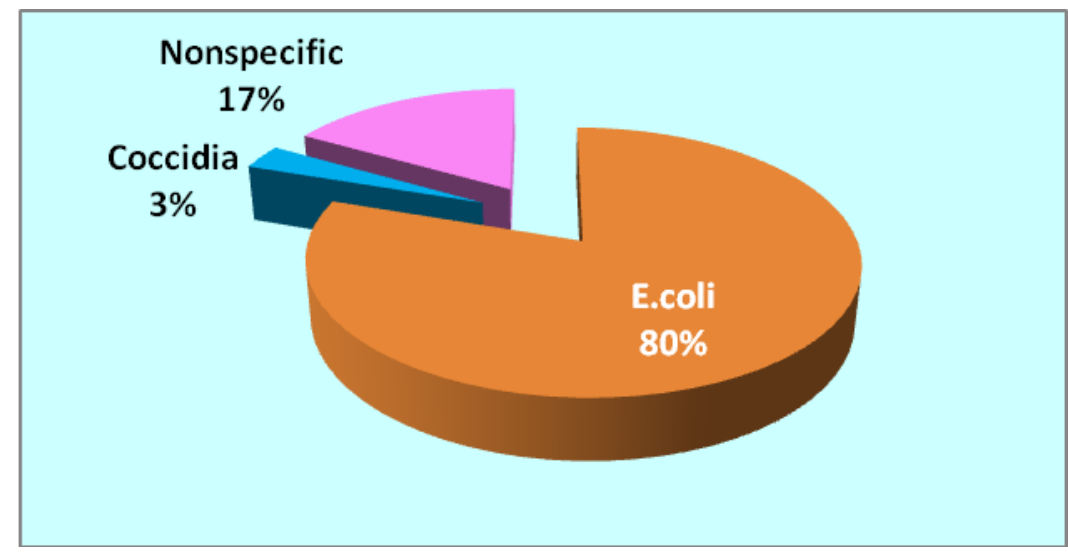

Fig.4 Antibiotic sensitivity test using discs

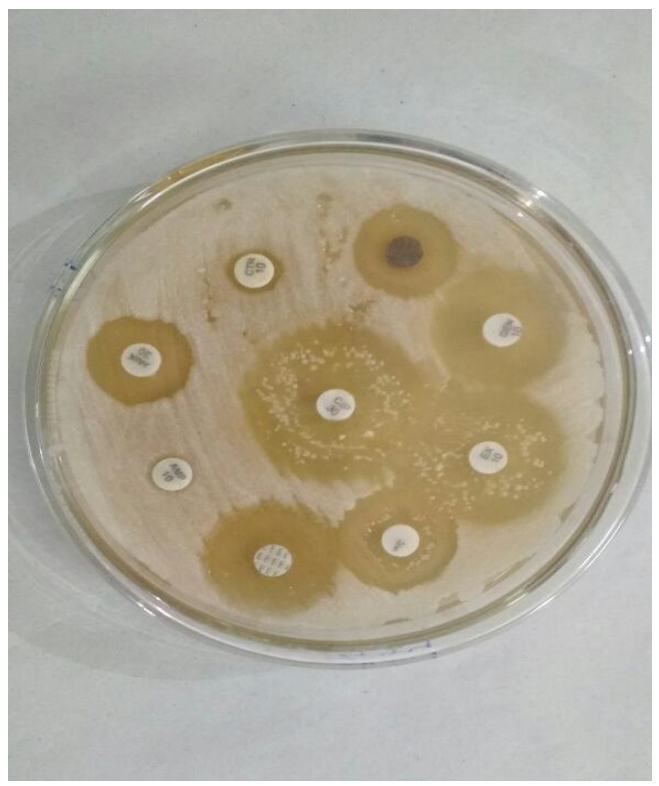

\section{Microbiological studies}

On microscopic examination of the Gram stained impression smears of intestinal luminal surface revealed presence of large number of Gram negative (pink colour) small rods arranged in singe, pair or in short chains. E. coli were isolated from the intestinal swabs by routine procedure on eosin Methylene Blue agar with a metallic sheen over the surface of the growth media so also on MacConkey's Lactose Agar there was presence of pink colour colonies. Zinnah et al., (2007) studied and observed all the E. coli isolates which produced bright pink colonies on MacConkey agar, yellowish green colonies surrounded by an intense yellow green zone on Brilliant Green agar and characteristic metallic sheen colonies on the Eosin Methylene Blue agar. In Gram's staining technique, all the isolates were pink colored, small rod shaped Gram negative bacilli. Roshid et al., (2013) detected and characterized $E$. coli from gut, liver and lungs 
of day old chickens. They observed black colour colony with metallic sheen in EMB media, bright pink colored smooth colonies in MC agar and pink colored smooth colonies in SS agar as a cultural characteristics arranged in single or chain as morphological characteristics. Tonu et al., (2011) examined all 10 isolates after Gram's staining which revealed Gram-negative, pink coloured, short rod shaped organisms arranged as single or in pair. The morphology of the isolated bacteria from 10 isolates exhibited Gram negative, pink color, short rod to coccoid shape. Chotiah and Damayanti (2017) isolated $76.02 \%$ E. coli from a total of 196 samples collected from intestine, liver, heart, egg yolk, air sac and faeces.

\section{Antibiotic sensitivity test}

The antibiotic sensitivity test of avian colibacillosis showed highest sensitivity for Gentamicin (90\%) followed by Amikacin (80\%), Colistin (70\%), Cefotaxime (60\%), Enrofloxacin (50\%), Ciprofloxacin (40\%), Ampicillin (20\%), Chloramphenicol (20\%) and Lyncomycin (10\%) respectively (Table 2 and Fig. 4). Saidi et al (2016) showed that there were high prevalence of sensitivity of avian pathogenic E. coli (APEC) to ciprofloxacin (100\%) and gentamycin $(97.1 \%)$. The isolates showed moderate rates of sensitivity to chloramphenicol and neomycin in Harare, Zimbabwe. Chotiah and Damayanti (2017) isolated E. coli from septicaemia, sick and dead chickens collected from three districts in West Java District showing E.coliis sensitive to chloramphenicol and resistant to ampicillin, neomycin and sulfamethoxazole,

trimethoprim. Antibiogram profiles indicated that gentamycin sensitivity was $64.5 \%$.

It was concluded that prevalence of enteritis in different farms was more during rainy than summer and winter seasons. The prevalence of enteritis in different farms revealed that birds of 0-14 week's age group (51\%) were more susceptible to the disease than older age groups.It was revealed by etiological diagnosis of enteritis that $E$. coli has the highest involvement $(80 \%)$ than nonspecific causes $(17 \%)$ and coccidiosis (3\%).The average values of heterophils \%, and TLC values were higher whereas average value of lymphocyte and monocytes, were significantly decreased in the affected birds in comparison to that of apparently healthy birds. The antibiotic which showed highest sensitivity for aviancolibacillosis was Gentamicin.

\section{Acknowledgement}

The authors are grateful to the Dean, Orissa University of Agriculture and Technology, Bhubaneswar, Odisha for providing the facilities to carry out this research work.

\section{References}

Arukha, P. 2015.Pathology of bacterial enteritis in chicken. M.V.Sc. Thesis submitted to Orissa University of Agriculture and Technology, Bhubaneswar, Odisha

Balasubramaniam, A., Dorairajan, N. 2009. Influence of season on occurrence of poultry diseases inNamakkal region of South India. The Indian Journal of Field Veterinarians. 4(4): 27-29.

Bhalerao, A.K.D., Gupta, R.P and Mamta, K. 2013. Pathological studies on natural cases of avian, colibacillosis in Haryana state. Haryana Veterinarian. 52: 118-120.

Bonia, R., Phangcho, C.V., Mukit, A., Saikia, G.K. 2010. Incidence and pathological conditions in chicken of Kalinga Brown breed in Guwahati, Assam, Indian Journal of Veterinary Pathology. 34(1):43-45.

Chotiah, S and Damayanti,R.2017 Colibacillosis and Antibiotics Resistance Patterns in Broiler. Proceedings of International Seminar of LPVT-2016.p.434-440.

DattaS,Rakha, N.K,Narang, G and Mahajan, N.K. 
2013. Epidemiology of enteritis in broiler chickens in the state Of Haryana and its association with different Meteorological parameters. Haryana Veterinarian.52: 22-25.

Guabiraba, R and Schouler, C. 2015. Avian colibacillosis: still many black holes. FEMS Microbiol Lett, Volume 362, Issue 15, 1 August 2015, fnv118,

Hermans, P.G and Morgan, K.L. 2007. Prevalence and associated risk factors of necrotic enteritis on broiler farms in the United Kingdom; a cross-sectional survey.Avian Pathology. 36(1): 43-51.

Islam, M.N., Rashid, S.M.H., Juli, M.S.B., Hoque, M.F and Akter, M.R. 2009. Necrotic enteritis in chickens: pathological, bacteriological and therapeutical investigation, International Journal on Sustainable Crop Production, 4(3): 1-7.

Islam, M.T, Islam, M.A, Samad, M.A and Kabir, S.M.L.2004. Characterization and antibiogram of $E$. coli associated with mortality in broilers and ducklings in Bangladesh.Bangladesh Journal of Veterinary Medicine. 2(1): 09-14.

Kabir, S.M.L. 2010. Avian Colibacillosis and Salmonellosis: A Closer Look at Epidemiology, Pathogenesis, Diagnosis, Control and Public Health Concerns. International Journal of Environmental Research and Public Health. 7: 89-114.

Korver, D. R. 2006. Overview of the immune dynamics of the digestive system. The Journal of Applied Poultry Research.15:123135.

Binek M., Borzemska W., Pisarski R., Błaszczak B., Kosowska G., Malec H., Karpińska E. 2000. Evaluation of the efficacy of feed providing on development of gastrointestinal microflora of newly hatched broiler chickens. European Poultry Science.64:147-
151.

Mishra, H.S. 2014.Clinicopathological studies on heat stress in chicken. M.V.Sc. Thesis submitted to Orissa University of Agriculture and Technology, Bhubaneswar, Odisha

Rahman, M.A, Samad, M.A, Rahman M.B, Kabir S.M.L. 2004. Bacteriopathology of salmonellosis, collibacillosis, pasteurellosis in natural and experimental infections in chickens.Bangladesh Journal of Veterinary Medicine. 2(1): 1-8.

Rashid,M.A., Chowdhury, K.A., Mahmud, S.M.S, Chowdhury, T., Bhuyan, A.A.M, Nahar, Z. and Paul, S.K.2013. E. coli from day old chicks of a selected breeder farm in Bangladesh. Vetscan. 7(2): 36-39.

Saidi, B., Mafirakureva, P., Mbanga, J. 2016. Antimicrobial resistance of $E$. coli isolated from chickens with colibacillosis in and around Harare, Zimbabwe. Proceedings of International Seminar of LPVT-2016.p.434440.

Tonu, N.S., Sufian, M.A., Sarker, S., Kamal, M.M., Rahman, M.H. and Hossain, M.M. 2011.Pathological study on colibacillosis in chickens and detection of E. coli by PCR. Bangladesh Journal of Veterinary Medicine. 9(1): $17-25$.

Wayne, P.A.2011. Performance standards for antimicrobial susceptibility testing. TwentyFirst Informational Supplement. CLSI document M100-S21.

Zinnah A.M .2007. Characterization and drug sensitivity pattern of $E$. coli isolated from different biological and environmental sources. M.S. Thesis, Department of Microbiology and Hygiene, BAU, Mymensingh.p.20.

\section{How to cite this article:}

Mayadevi Majhi, Jasmine Pamia, Susen Kumar Panda, Lipismita Samal and Rajashree Mishra. 2018. Effect of Age and Season on Enteritis and Antibiotic Sensitivity Test of E. coli Isolated from Infected Chickens in Odisha, India. Int.J.Curr.Microbiol.App.Sci. 7(03): 2037-2045. doi: https://doi.org/10.20546/ijcmas.2018.703.239 